\title{
Novel Exact Solitary Wave Solutions for the Time Fractional Generalized Hirota-Satsuma Coupled KdV Model Through the Generalized Kudryshov Method
}

\author{
1,2,* Mohammad Safi Ullah, ${ }^{2,3}$ Harun-Or-Roshid, ${ }^{2}$ M. Zulfikar Ali and ${ }^{1,2}$ Zillur Rahman \\ ${ }^{1}$ Department of Mathematics, Comilla University, Cumilla-3506, Bangladesh \\ ${ }^{2}$ Department of Mathematics, Rajshahi University, Rajshahi-6205, Bangladesh \\ ${ }^{3}$ Department of Mathematics, Pabna University of Science and Technology, Pabna-6600, Bangladesh \\ Email: safi.ru1985@gmail.com and Email: harunorroshidmd@gmail.com
}

\begin{abstract}
In the current article, the generalized Kudryshov method is applied to determine exact solitary wave solutions for the time-fractional generalized Hirota-Satsuma coupled $\mathrm{KdV}$ model. Here, the fractional derivative is illustrated in the conformable derivative. Therefore, plentiful exact traveling wave solutions are achieved for this model, which encourage us to enlarge, a novel technique to gain unsteady solutions of autonomous nonlinear evolution models that occurs in physical and engineering branches. The obtained traveling wave solutions are expressed in terms of the exponential and rational functions. It is effortless to widen that this method is powerful and will be applied in further tasks to create advance exclusively innovative solutions to other higher-order nonlinear conformable fractional differential models in engineering problems.
\end{abstract}

Keywords: the generalized Kudryshov method; exact solitary wave solutions; time-fractional generalized Hirota-Satsuma coupled (HSC) KdV system; conformable fractional derivative

\section{Introduction}

Nearby, great interest in fractional calculus applied in various fields such as electrical networks, control theory of dynamics, statistics, electro-chemistry of oxidization, acoustics, nonlinear optical fiber, plasma and solid-state physics, chemical kinetics and geochemistry phenomena, signal processing, and data mining can be effectively formed by means of nonlinear fractional-order differential systems ${ }^{[1-6] .}$ Modeling of a range of physical phenomena in terms of nonlinear timefractional evolution equations has played a significant factor in numerous efficient applications in the above mentioned fields.

The time-fractional generalized HSC KdV system is a vital nonlinear model that occurs in the Toda lattice equation, a recognized $(1+1)$ dimension soliton equation. This system can also be utilized as the model of interaction of neighboring particles of the same mass in a lattice formation with a crystal as well as illustrated basic characteristics of string dynamics in constant curvature space ${ }^{[13]}$.

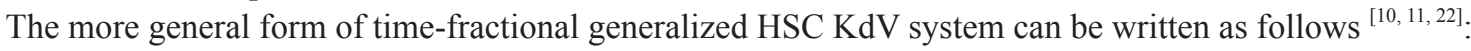

$$
\left\{\begin{array}{l}
\frac{\partial^{\alpha} h}{\partial t^{\alpha}}=\frac{1}{4} h_{x x x}+3 h h_{x}+3\left(w-v^{2}\right)_{x} \\
\frac{\partial^{\alpha} v}{\partial t^{\alpha}}=-\frac{1}{2} v_{x x x}-3 h v_{x}, \quad 0<\alpha \leq 1 \\
\frac{\partial^{\alpha} w}{\partial t^{\alpha}}=-\frac{1}{2} w_{x x x}-3 h w_{x}
\end{array}\right.
$$

Recently, searching exact solutions of the system Eq.(1) was found by renowned researchers ${ }^{[12,13]}$.Guo et. al. ${ }^{[12]}$ 
applied the improved fractional sub-equation method to construct analytical solutions to the space-time-fractional equations arising in fluid mechanics. The exact and complex traveling wave solutions to the time-fractional generalized Hirota-Satsuma coupled KdV system are deliberated by Neirameh ${ }^{[13]}$ using the direct algebraic method.

Considerable effort has been paid by many dynamical researchers to investigate exact solutions for FDEs such as the impulsive fractional differential equations with different boundary conditions ${ }^{[14,15,16]}$, nonlinear impulsive hybrid boundary value problems involving fractional differential equations ${ }^{[17]}$, Space-Time-fractional Burgers equation ${ }^{[18]}$, time-fractional Burgers equation in fluid flow ${ }^{[19],}$ the fractional coupled viscous Burgers' equation ${ }^{[20]}$, Time-fractional KdV equations ${ }^{[21]}$ and so on.

The objective of this paper is to apply the generalized Kudryashov method for finding the exact solitary wave solutions of the time-fractional generalized HSC KdV system, which take part in a key task in mathematical physics.

This paper is organized as follows: fundamental properties of the conformable fractional derivative are presented in section 2. A brief description of the generalized Kudryashov methods is given in section 3. Then in section 4, this method has been applied to establish exact solutions for the time-fractional general HSC KdV system. The obtained results are presented graphically and the relevant physical illustrations are provided in section 5. Finally, concluding remarks are drawn in section 6 .

\section{Conformable fractional derivative and its properties}

For a function $\phi:(0, \infty) \rightarrow \mathfrak{R}$, the conformable fractional derivative of $\phi$ for order $\alpha$ is defined ${ }^{[23]}$ as

$$
\frac{\partial^{\alpha} \phi}{\partial t^{\alpha}}=\lim _{\varepsilon \rightarrow 0^{+}} \frac{\phi\left(t+\varepsilon t^{1-\alpha}\right)-\phi(t)}{\varepsilon}, t>0 \quad \text { and } \quad 0<\alpha \leq 1
$$

Some important properties of the conformable fractional derivative are as follows:

$$
\begin{aligned}
& \frac{\partial^{\alpha}}{\partial t^{\alpha}}(a \phi+b \varphi)=a \frac{\partial^{\alpha}}{\partial t^{\alpha}}(\phi)+b \frac{\partial^{\alpha}}{\partial t^{\alpha}}(\varphi), \forall a, b \in \Re . \\
& \frac{\partial^{\alpha}}{\partial t^{\alpha}}\left(t^{\beta}\right)=\beta t^{\beta-\alpha}, \forall \beta \in \Re \text { and } \frac{\partial^{\alpha}}{\partial t^{\alpha}}(\lambda)=0, \lambda=\text { const. } \\
& \frac{\partial^{\alpha}}{\partial t^{\alpha}}(\phi \circ \varphi)(t)=t^{1-\alpha} \phi^{\prime}(\varphi(t)) \varphi^{\prime}(t) .
\end{aligned}
$$

\section{The Method}

Let us assume a general nonlinear evolution equation in $x$ and $t$ as

$$
\aleph\left(h, h_{t}, h_{x}, h_{x}, \ldots\right)=0, x \in \mathfrak{R}, t>0,
$$

where the function $h=h(x, t)$ is unknown and $\aleph$ is a polynomial function with respect to some functions or specified variables, which have nonlinear terms and highest order derivatives of the unknown function. The key steps of the generalized Kudryashov method are as ${ }^{[7,9,21]}$ :

Step 1: Consider the following traveling wave transformation

$$
h(x, t)=H(\zeta), \zeta=x-\frac{c t^{\alpha}}{\alpha}
$$

where $c$ is the velocity of the relative wave mode. By using the above transformation the nonlinear partial differential equation (3) is reduced to a nonlinear ordinary differential equation (ODE)

$$
\chi\left(H, H^{\prime}, H^{\prime \prime}, \ldots\right)=0,
$$


where the prime denotes the derivative of $H$ with respect to $\zeta$ and $\chi$ is a polynomial of $H(\zeta)$.

Step 2: Let us assume that the solution of Eq. (4) has the following form:

$$
H(\zeta)=\frac{\sum_{i=0}^{N} a_{i} \phi^{i}(\zeta)}{\sum_{j=0}^{M} b_{j} \phi^{j}(\zeta)}
$$

where $a_{i}$ and $b_{j}$ are real constants, $N$ and $M$ are positive integers such that $a_{N}, b_{M} \neq 0$ and $\phi(\zeta)$ satisfies the following ordinary differential equation:

$$
\phi^{\prime}(\zeta)=\phi^{2}(\zeta)-\phi(\zeta)
$$

The general solution of Eq. (6) is of the following form:

$$
\phi(\zeta)=\frac{1}{1+A e^{\zeta}}
$$

where $A$ is any arbitrary constant.

Step 3: Determine the positive integers $N$ and $M$ in Eq. (5) by balancing the highest order derivative term with the nonlinear term of $H(\zeta)$ in Eq. (2) or Eq. (4). Moreover, we define the degree of $H(\zeta)$ as $D(H(\zeta)=N-M$, which gives rise to the degree of other expressions as

$$
D\left(\frac{d^{q} H}{d \zeta^{q}}\right)=N-M+q, D\left(H^{p}\left(\frac{d^{q} H}{d \zeta^{q}}\right)^{s}\right)=(N-M) p+s(N-M+q),
$$

where $p, q, s$ are integer numbers.

Thus, we can find the value of $N$ and $M$ in Eq. (5).

Step 4: Insert Eq. (5) along with Eq. (6) into Eq. (4) and collect all terms with the same powers of $\phi$ together. Setting each coefficient of this polynomial $\phi$ to zero, we obtain a system of algebraic equations for $a_{i}, b_{j}$ and $c$.

Step 5: By inserting the values of parameters gained in the previous step and $\phi(\zeta)$ into the Eq. (5), then the solutions of Eq. (2) can be constructed.

\section{Applications}

Consider the following traveling wave transformation:

$$
\left\{\begin{array}{l}
h(x, t)=\frac{1}{c} H^{2}(\xi) \\
v(x, t)=-c+H(\xi) \\
w(x, t)=2 c^{2}-2 c H(\xi)
\end{array}\right.
$$

where $\xi=x-\frac{c}{\alpha} \cdot t^{\alpha}$.

Inserting Eq. (8) into Eq. (1) reduced into ordinary differential equations

$$
c\left(H_{\xi}\right)^{2}+c H H_{\xi}+3 H^{4}-4 c^{2} H^{2}+6 c^{4}+2 c^{2} R=0
$$


and $c H_{\xi \xi}+2 H^{3}-2 c^{2} H=0$.

where $R$ is an integration constant to be evaluated later.

Case 1: By balancing the highest order derivative term $H H_{\text {sg }}$ with the nonlinear term $H^{4}$ in Eq. (9) gives $N=M+1$

Setting $M=1$, we have $N=2$. Therefore Eq. (5) reduces to

$$
H(\xi)=\frac{a_{0}+a_{1} \phi+a_{2} \phi^{2}}{b_{0}+b_{1} \phi}
$$

Inserting Eq.(11) along with Eq.(6) into Eq.(9), we have a polynomial of $\phi^{k},(k=0,1,2, \ldots)$. Equating the coefficients of this polynomial of the same powers of $\phi$ to zero, we obtain a system of equations yields the values for $R, c$, $a_{0}, a_{1}, a_{2}, b_{0}$ and $b_{1}$.

Set 1: $R=-\frac{5}{32}, c=-\frac{1}{4}, a_{0}=-\frac{1}{4} b_{0}, a_{1}=-\frac{1}{4} b_{1}+\frac{1}{2} b_{0}, a_{2}=\frac{1}{2} b_{1}, b_{0}, b_{1}=$ const.

Set 2: $R=-\frac{5}{2}, c=-1, a_{0}=\frac{1}{2} b_{1}, a_{1}=-b_{1}, a_{2}=b_{1}, b_{0}=-\frac{1}{2} b_{1}, b_{1}=$ const.

Set 3: $R=-\frac{5}{2}, c=-1, a_{0}=-\frac{1}{2} b_{1}, a_{1}=b_{1}, a_{2}=-b_{1}, b_{0}=-\frac{1}{2} b_{1}, b_{1}=$ const .

Set 4: $R=-\frac{5}{32}, c=-\frac{1}{4}, a_{0}=\frac{1}{4} b_{0}, a_{1}=\frac{1}{4} b_{1}-\frac{1}{2} b_{0}, a_{2}=-\frac{1}{2} b_{1}, b_{0}, b_{1}=$ const.

Set 5: $R=-\frac{3}{4}, c=\frac{1}{2}, a_{0}=0, a_{1}=\mp \frac{i}{\sqrt{2}} b_{1}, a_{2}=\mp \frac{i}{\sqrt{2}} b_{1}, b_{0}=-\frac{1}{2} b_{1}, b_{1}=$ const.

For set 1, the time-fractional generalized HSC KdV equations hold the solution as:

$\left\{\begin{array}{l}h(x, t)=-\frac{1}{4}\left\{\frac{1-A e^{\xi}}{1+A e^{\xi}}\right\}^{2} \\ v(x, t)=\frac{1}{2\left(1+A e^{\xi}\right)} \\ w(x, t)=\frac{1}{4\left(1+A e^{\xi}\right)}\end{array}\right.$,

where $\xi=x+\frac{t^{\alpha}}{4 \alpha}$

For set 2, the time-fractional generalized HSC KdV equations hold the solution as:

$$
\left\{\begin{array}{l}
h(x, t)=-\left\{\frac{1+A^{2} e^{2 \xi}}{1-A^{2} e^{2 \xi}}\right\}^{2} \\
v(x, t)=\frac{2}{1-A^{2} e^{2 \xi}} \\
w(x, t)=\frac{4}{1-A^{2} e^{2 \xi}}
\end{array}\right.
$$


where $\xi=x+\frac{t^{\alpha}}{\alpha}$

For set 3, the time-fractional generalized HSC KdV equations hold the solution as:

$$
\left\{\begin{array}{c}
h(x, t)=-\left\{\frac{1+A^{2} e^{2 \xi}}{1-A^{2} e^{2 \xi}}\right\}^{2} \\
v(x, t)=-\frac{2 A^{2} e^{2 \xi}}{1-A^{2} e^{2 \xi}} \\
w(x, t)=-\frac{4 A^{2} e^{2 \xi}}{1-A^{2} e^{2 \xi}}
\end{array}\right.
$$

where $\xi=x+\frac{t^{\alpha}}{\alpha}$

For set 4, the time-fractional generalized HSC KdV equations hold the solution as:

$$
\left\{\begin{array}{l}
h(x, t)=-\frac{1}{4}\left\{\frac{1-A e^{\xi}}{1+A e^{\xi}}\right\}^{2} \\
v(x, t)=\frac{A e^{\xi}}{2\left(1+A e^{\xi}\right)} \\
w(x, t)=\frac{A e^{\xi}}{4\left(1+A e^{\xi}\right)}
\end{array}\right.
$$

where $\xi=x+\frac{t^{\alpha}}{4 \alpha}$.

For set 5, the time-fractional generalized HSC KdV equations hold the solution as:

$$
\begin{aligned}
& \left\{\begin{array}{l}
h(x, t)=-4\left\{\frac{ \pm\left(1+A e^{\xi}\right) \mp 1}{1+A^{2} e^{2 \xi}}\right\}^{2} \\
v(x, t)=\frac{-\left(1-A^{2} e^{2 \xi}\right) \mp i 2 \sqrt{2}\left(1+A e^{\xi}\right) \pm i 2 \sqrt{2}}{2\left(1-A^{2} e^{2 \xi}\right)}, \\
w(x, t)=\frac{1-A^{2} e^{2 \xi} \mp i 2 \sqrt{2}\left(1+A e^{\xi}\right) \pm i 2 \sqrt{2}}{1-A^{2} e^{2 \xi}}
\end{array}\right. \\
& \text { where } \xi=x-\frac{t^{\alpha}}{2 \alpha} \text {. }
\end{aligned}
$$

Case 2: Balancing $H H_{\xi \xi}$ with $H^{4}$ in equation (10) gives $N=M+1$, Setting $M=1$, we obtain $N=2$. Therefore Eq. (5) reduces to

$$
H(\xi)=\frac{a_{0}+a_{1} \phi+a_{2} \phi^{2}}{b_{0}+b_{1} \phi}
$$

Inserting Eq.(17) along with Eq.(5) into Eq.(10), we get a polynomial of $\phi^{k},(k=0,1,2, \ldots)$. Equating the 
coefficients of this polynomial of the same powers of $\phi$ to zero, we obtain a system of equations yields the values forc, $a_{0}$, $a_{1}, a_{2}, b_{0}$ and $b_{1}$.

Set 1: $c=-\frac{1}{4}, a_{0}=-\frac{1}{4} b_{0}, a_{1}=-\frac{1}{4} b_{1}+\frac{1}{2} b_{0}, a_{2}=\frac{1}{2} b_{1}, b_{0}, b_{1}=$ const.

Set 2: $c=-1, a_{0}=\frac{1}{2} b_{1}, a_{1}=-b_{1}, a_{2}=b_{1}, b_{0}=-\frac{1}{2} b_{1}, b_{1}=$ const.

Set 3: $c=-1, a_{0}=-\frac{1}{2} b_{1}, a_{1}=b_{1}, a_{2}=-b_{1}, b_{0}=-\frac{1}{2} b_{1}, b_{1}=$ const.

Set 4: $c=-\frac{1}{4}, a_{0}=\frac{1}{4} b_{0}, a_{1}=\frac{1}{4} b_{1}-\frac{1}{2} b_{0}, a_{2}=-\frac{1}{2} b_{1}, b_{0}, b_{1}=$ const.

Set 5: $c=\frac{1}{2}, a_{0}=0, a_{1}= \pm i \sqrt{2} b_{0}, a_{2}=\mp i \sqrt{2} b_{0}, b_{0}=$ const, $b_{1}=2 b_{0}$.

From some simplification, we see that the set- 1 to set- 4 gives the same results as in case- 1 . But only set- 5 is different which gives the solution as

$$
\left\{\begin{array}{l}
h(x, t)=-4\left\{\frac{ \pm\left(1+A e^{\xi}\right) \mp 1}{-1+A^{2} e^{2 \xi}}\right\}^{2} \\
v(x, t)=\frac{1-A^{2} e^{2 \xi} \pm i 2 \sqrt{2}\left(1+A e^{\xi}\right) \mp i 2 \sqrt{2}}{2\left(-1+A^{2} e^{2 \xi}\right)} \\
w(x, t)=\frac{-1+A^{2} e^{2 \xi} \mp i \sqrt{2}\left(1+A e^{\xi}\right) \pm i \sqrt{2}}{2\left(-1+A^{2} e^{2 \xi}\right)}
\end{array}\right.
$$

where $\xi=x-\frac{t^{\alpha}}{2 \alpha}$.

\section{Graphical representations}

Ten sets of results are achieved in this research. All of the results are analyzed and some of them are depicted in the Figs. 1-2. The graphs signify the change of amplitude, the shape of the wave, and the nature of the solitary waves for each acquired wave solutions in space $\mathrm{x}$ at time t. The solution $h(x, t)$ of Eq.(12) represents bright bell solitary wave (Fig-1(a)) for the physical parameters $A=0.5, \alpha=0.67$ The solution both $v(x, t)$ and $w(x, t)$ of Eq.(12) represents similar kink solitary wave. Fig-1(b) expressed the shape of the kink wave $v(x, t)$ of Eq.(12) for the physical parameters $A=0.5, \alpha=0.67$.

The solutions $h(x, t)$ of Eq.(15) represent bright bell solitary wave solutions and all of them are similar to the graph Fig-1(a) of $h(x, t)$ in Eq.(12). We also see that the solutions $v(x, t), w(x, t)$ of Eq.(15) represent kink solitary wave solutions and all of them are similar to the graph Fig-1(b) of $h(x, t)$ in Eq.(12). So we omit the similar type of figures.

The solution $h(x, t)$ of Eq.(13) represents dark solitary wave (see Fig-2(a)) for the physical parameters $A=0.5, \alpha=0.5$ in space $\mathrm{x}$ at time t. The solution both $v(x, t)$ and $w(x, t)$ of Eq.(13) represents a similar singular kink solitary wave. Fig-2(b) expressed the shape of the singular kink solitary wave $v(x, t)$ of Eq.(13) for the physical parameters $A=0.5, \alpha=0.5$.

The solutions $h(x, t)$ of Eq.(14), Eq.(16), and Eq.(18) represent dark bell solitary wave solutions and all of them are similar to the graph Fig-2(a) of $h(x, t)$ in Eq.(13). We also see that the solutions $v(x, t), w(x, t)$ of Eq.(14) and complex part of $v(x, t), w(x, t)$ of Eq. $(16)$ and $v(x, t), w(x, t)$ of Eq.(18) represent singular kink solitary wave solutions and all of them are similar like to the graph Fig-2(b) of $h(x, t)$ in Eq.(13). So we omit the similar type of figures. The real part of in Eq.(16) and Eq.(18) gives constant solution that represent in $x t$ plane. 


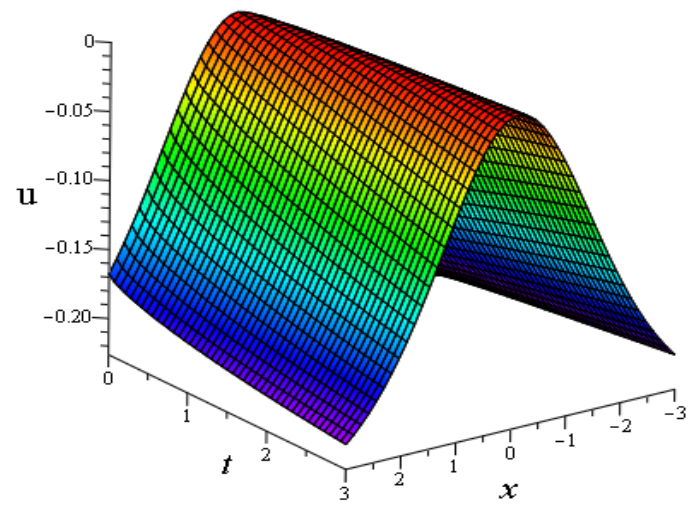

(a)

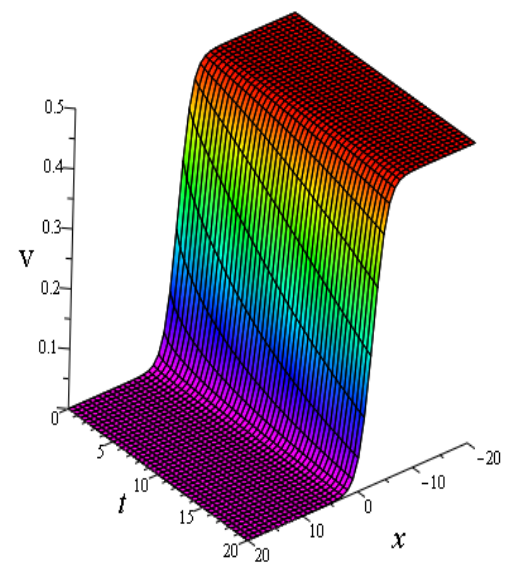

(b)

Figure 1 (a) Represent bright bell solitary wave solution $h(x, t)$ of Eq.(12) for the physical parametric values $A=0.5, \alpha=0.67$ and (b) Represent kink solitary wave solution $v(x, t)$ of Eq.(12) for the physical parametric values $A=0.5, \alpha=0.67$.

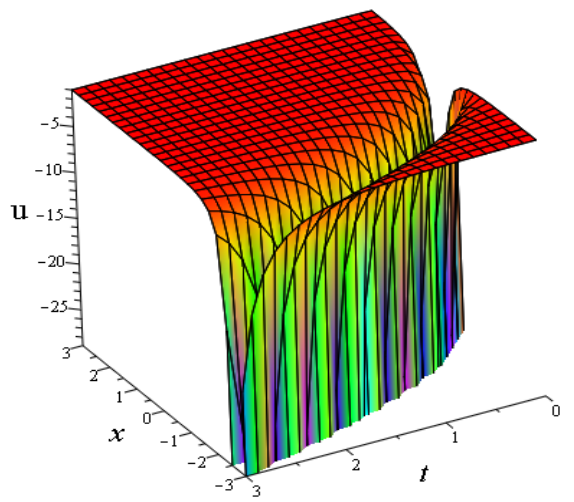

(a)

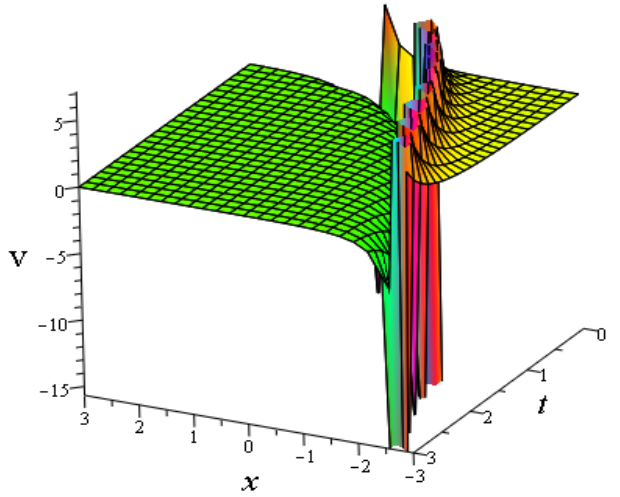

(b)

Figure 2 (a) Represent dark bell solitary wave solution $h(x, t)$ of Eq.(13) for the physical parametric values $A=0.5, \alpha=0.67$ and (b) Represent kink solitary wave solution $v(x, t)$ of Eq.(13) for the physical parametric values $A=0.5, \alpha=0.67$.

\section{Conclusions}

In this article, we have successfully used a mathematical apparatus named the generalized Kudryashov method for creating exact solitary wave solutions to the time-fractional generalized Hirota-Satsuma coupled KdV system. The achieved solitary wave solutions are expressed in terms of the exponential and rational functions. The acquired results will serve as a very important milestone in the study of the interaction of neighboring particles of the same mass in a lattice formation with a crystal and long water wave phenomena. We also have demonstrated that the generalized Kudryashov method is an effective solvable tool for large classes of system of conformable nonlinear fractional differential equations.

Conflict of Interest: The authors declare that they have no conflict of interest.

\section{References}

[1] K.B. Oldham, J. Spanier. The Fractional Calculus. New York: Academic Press; 1974.

[2] I. Podlubny. Numerical solution of ordinary fractional differential equations by the fractional difference method. In Elaydi, S., Gyori, I. and Ladas, G. (Eds.) Advances in Difference Equations. Amsterdam: Gordon and Breach; 1997.

[3] I. Podlubny. Fractional Differential Equations. New York: Academic Press; 1999. 
[4] S.G. Samko, A.A. Kilbas, O.I. Marichev. Fractional Integrals and Derivatives: Theory and Applications. Yverdon: Gordon and Breach; 1993.

[5] K.S. Miller, B. Ross. An Introduction to the Fractional Calculus and Fractional Differential Equations. New York: Wiley; 1993.

[6] M. Caputo. Linear models of dissipation whose Q is almost frequency independent. J. Roy. Astr. Soc., 1967; 13: 529539.

[7] M.R. Islam, H.O. Roshid. Application of Generalized Kudryashov Method to the Burger Equation. Int. J. Math. Trends and Tech. (IJMTT), 2017; 38(2): 111-113.

[8] M.J. Ablowitz, H. Segur. Solitons and Inverse Scattering Transform. Philadephia: SIAM; (1981).

[9] S.T. Demiray, Y. Pandir, H. Bulut. Generalized Kudryashov method for time-fractional differential equations. In: Abstract and applied analysis. 2014; 2014 (Article ID 901540).

[10] Z.Z. Ganji, D.D. Gangi, Y. Rostamiyan. Solitary wave solutions for a time-fraction generalized Hirota-Satsuma coupled KdV equation by an analytical technique. Appl. Math. Model. 2009; 33 (7): 3107-3113.

[11] M. Shateri, D.D. Ganji. Solitary Wave Solutions for a Time-Fraction Generalized Hirota-Satsuma Coupled KdV Equation by a New Analytical Technique. Int. J. Differ. Equ. 2010; 2010: 117-223. Article ID 954674.

[12] S. Guo, L. Mei , Y. Li , Y. Sun . The improved fractional sub-equation method and its applications to the space-time fractional differential equations in fluid mechanics. Phys. Lett. A 2012;376 (4): 407-411.

[13] A. Neirameh. Soliton Solutions of the Time Fractional Generalized Hirota-satsuma Coupled KdV System. Appl. Math. Inf. Sci. 2015; 9(4): 1847-1853.

[14] G.M. Mophou. Existence and uniqueness of mild solutions to impulsive fractional differential equations . Nonlinear Anal. 2010; 72: 1604-1615.

[15] G. Wang, B. Ahmad, L. Zhang, Some existence results for impulsive nonlinear fractional differential equations with mixed boundary conditions. Comput. Math. Appl. 2011; 62(3): 1379-1397.

[16] X. Zhang, X. Huang, Z. Liu. The existence and uniqueness of mild solutions for impulsive fractional equations with nonlocal conditions and infinite delay. Nonlinear Anal. Hybrid Syst.2010; 4: 775-781.

[17] B. Ahmad, S. Sivasundaram. Existence results for nonlinear impulsive hybrid boundary value problems involving fractional differential equations. Nonlinear Anal.: Hybrid Syst. 2009; 3: 251-258.

[18] A. B. Emad, Abdel-Salamand, J. H. Ahmed. Solutions of the $(2+1)$ Space-Time Fractional Burgers equation. American Journal of Computational and Applied Mathematics. 2016; 6(2): 109-117.

[19] A. Yokus, D. Kaya. Numerical and exact solutions for time fractional Burgers' equation. J. Nonlinear Sci. Appl. 2017; 10: 3419-3428.

[20] T. A. Sulaiman, M.Yavuz, H. Bulut, H. M. Baskonus. Investigation of the fractional coupled viscous Burgers' equation involving Mittag-Leffler kernel. Physica A: Statistical Mechanics and its Applications, 2019; 527(1): 121-126.

[21] H. Bulut, Y. Pandir, S. T. Demiray. Exact Solutions of Time-Fractional KdV Equations by Using Generalized Kudryashov Method. International Journal of Modeling and Optimization, 2014; 4(4): 315-320.

[22] A.S. Arife, S.K. Vanani, A. Yildirim. Numerical Solution of Hirota-Satsuma Couple Kdv and a Coupled MKdv Equation by Means of Homotopy Analysis Method. World Appl. Sci. J. 2011; 01(13): 2271-2276.

[23] R. Khalil , M.A. Horani , A. Yousef , M. Sababheh. A new definition of fractional derivative. Journal of Computational and Applied Mathematics 2014; 264: 65-70. 